\title{
APORTES EDUCATIVOS EN SALUD INTEGRAL EN MUJERES ADOLESCENTES
}

Educational contributions in integral health in adolescent women

Fecha de Recepción: 7 noviembre 2020
Contribuições educacionais na saúde integral da mulher adolescente

Aceptado para su publicación: 30 noviembre 2020

\section{Autores: \\ Horacio J Romero ${ }^{1, a}$ Eduardo A Achitte ${ }^{2, b}$ Silvia R Pérez ${ }^{3, c}$}

1. Área Disciplinar: Clínica Operatoria dental. Módulo Clínica Rehabilitadora I. Facultad de Odontología. Universidad Nacional del Nordeste. República Argentina.

2. Departamento Practica Final Obligatoria. Internado

Rotatorio y Pasantía Rural. Facultad de Medicina. Universidad Nacional del Nordeste. República Argentina.

3. Área Disciplinar: Práctica Clínica Preventiva I y II. Módulo Introducción a la Práctica Clínica. Facultad de

Odontología. Universidad Nacional del Nordeste. República Argentina.

a. Doctor de la Universidad Nacional del Nordeste en Odontología (UNNE)

b. Especialista en Diagnóstico por Imágenes (Universidad

Maimónides). Especialista en Obstetricia (UNNE)

c. Especialista en Metodología de la Investigación en Ciencias de la Salud (UNNE). Especialista en Docencia Universitaria (UNNE)

Correspondencia:

Romero, Horacio

Facultad de Odontología UNNE | Av. Libertad 5450, W3400 Corrientes, Argentina +540379 445-7992

Correo electrónico: hjromero@odn.unne.edu.ar hjromero18@yahoo.com.ar

Conflicto de intereses: los autores declaran no tener conflictos de interés.

Fuente de financiamiento: Programa Universidad en el Medio. Secretaría General de Extensión Universitaria.

\section{Resumen}

Las actividades de extensión desarrolladas en la comunidad nos permiten llegar a un gran número de personas con alta vulnerabilidad. El objetivo de este trabajo de extensión fue lograr una mejor calidad de vida tratando en forma integral la salud de un grupo de mujeres adolescentes en estado de vulnerabilidad. Basamos nuestra labor en la motivación hacia el cuidado de la salud en general y la salud bucodental a través de charlas informativas y acciones preventivas donde se trabajó sobre factores que interfieren el equilibrio del estado de salud. Charlas educativas, talleres sobre educación sexual, prevención de enfermedades sexuales y métodos de cuidado, respaldado en leyes nacionales vigentes. Los resultados fueron satisfactorios, se logró despertar el interés, motivación y acciones de mejoramiento en hábitos de higiene bucal, logrando niveles compatibles con salud en un $80 \%$ de las adolescentes intervenidas. Relacionar estas manifestaciones bucales, con las de salud general brindó herramientas para el autocuidado de mujeres adolescentes.

Palabras clave: salud de la mujer, adolescentes, vulnerabilidad en salud, relaciones comunidad-institución (fuente: DeCS BIREME).

\footnotetext{
Abstract

The extension activities developed in the community allow us to reach a large number of people with high vulnerability. The objective of this extension work was to achieve a better quality of life by comprehensively treating the health of a group of adolescent women in a state of vulnerability. We base our work on the motivation towards health care in general and oral health through informative talks and preventive actions in which we worked on factors that interfere with the balance of health status. Educational talks, workshops
} 
on sexual education, prevention of sexual diseases and methods of care, supported by current national laws. The results were satisfactory; we were able to awaken interest, motivation and actions to improve oral hygiene habits, achieving levels compatible with health in $80 \%$ of the intervened adolescents. Relating these oral manifestations to those of general health provided tools for the self-care of adolescent women.

Key words: women's health, adolescents, health vulnerability, community-institutional relations (source: MeSH NLM).

\section{Resumo}

As atividades de extensão realizadas na comunidade nos permitem alcançar um grande número de pessoas altamente vulneráveis. O objetivo deste trabalho de extensão foi alcançar uma melhor qualidade de vida por meio do atendimento integral à saúde de um grupo de adolescentes em situação de vulnerabilidade. Baseamos nosso trabalho na motivação para a atenção à saúde em geral e saúde bucal por meio de palestras informativas $e$ ações preventivas onde trabalhamos os fatores que interferem no equilíbrio do estado de saúde. Palestras educativas, oficinas de educação sexual, prevenção de doenças sexuais e métodos de atendimento, amparadas pela legislação nacional vigente. Os resultados foram satisfatórios, foi possivel despertar interesse, motivação e ações de melhoria nos hábitos de higiene bucal, atingindo níveis compatíveis com a saúde em $80 \%$ dos adolescentes intervencionados. Relacionar essas manifestações bucais com as de saúde geral forneceu ferramentas para o autocuidado de mulheres adolescentes.

Palavras-chave: saúde da mulher, adolescentes, vulnerabilidade em saúde, relações comunidade-instituição (fonte: DeCS BIREME).

\section{Introducción}

La promoción de prácticas saludables en la adolescencia y la adopción de medidas para proteger a los jóvenes frente a los riesgos para su salud, son fundamentales para prevenir la aparición de problemas de salud en la edad adulta ${ }^{1}$.

La mayoría de los jóvenes están sanos. Sin embargo, cada año se registran más de 2,6 millones de defunciones en la población de 15 a 24 años. En esta etapa se dan con mayor intensidad conductas de riesgo, que pueden llevar a consecuencias nocivas, entre ellas el consumo de tabaco, la falta de actividad física, las relaciones sexuales sin protección y la exposición a la violencia ${ }^{2,3}$.

Los adolescentes son uno de los grupos de mayor riesgo de afecciones bucales, debido a que atraviesan por una serie de cambios, que, aunados a los cambios hormonales durante la pubertad, crecimiento maxilar, maloclusiones, malos hábitos, convierten la atención en un reto. La mayoría de los jóvenes están más interesados en lucir una sonrisa blanca, dando mayor importancia a la estética ${ }^{4,5}$.

Las acciones de prevención y promoción de la salud son prioritarias en los grupos de alta vulnerabilidad. Es importante concientizar a los adolescentes sobre buenos hábitos de cuidado bucal y general, e incorporar la visita al profesional de la salud en forma periódica ${ }^{6-8}$

\section{Objetivos}

El objetivo general fue identificar las necesidades de prevención, promoción, educación e intervención en forma integral de la salud en mujeres adolescentes que asisten al comedor Pocho Roch del Barrio Quilmes y Centro Recreativo Los de Inmaguaré de la localidad de Riachuelo.

Los objetivos específicos fueron: 1) identificar las necesidades de tratamiento preventivo médico y odontológico, 2) aconsejar sobre los cuidados con respecto a la higiene personal de las adolescentes, 3) asesorar sobre hábitos de alimentación, identificando a los alimentos perjudiciales para la salud y como sustituirlos por una dieta saludable, 4) establecer estrategias de abordaje sobre educación sexual previniendo enfermedades y embarazo adolescente, 5) indicar hábitos de higiene bucal a través de la correcta técnica de cepillado, 6) trabajar en prevención y promoción de enfermedades bucales, tratando a la enfermedad en sus primeros estadios.

\section{Metodología}

El proyecto estuvo destinado a mujeres ado- 


\section{REFO}

VOL. XIII | N² 2| AÑO 2020

ISSN 1668-7280

ISSN-E 2683-7986 lescentes de 12 a 24 años de edad que asisten al comedor Pocho Roch del Barrio Quilmes y Centro Recreativo Los de Inmaguaré de la localidad de Riachuelo, de escasos recursos económicos, sin cobertura social, que los califica como de alto grado de vulnerabilidad biológica-social.

Las actividades se llevaron a cabo mediante reuniones del equipo de trabajo, organizadas en módulos, en forma secuencial y ordenada para su mejor desarrollo.

En el primer módulo, se brindó la información necesaria a las adolescentes sobre los siguientes temas: 1) hábitos de higiene, ¿Será necesario modificarlos?, 2) la alimentación, como lograr la dieta adecuada, 3) importancia del control periódico en el Centro de Salud más cercano.

En el segundo módulo se realizó el relevamiento de los siguientes datos: 1) número de embarazo en el grupo destinatario, 2) conocimiento de las enfermedades de transmisión sexual, 3) indicadores epidemiológicos a las adolescentes para saber el estado de salud bucal, mediante los índices Gingival de Loe Silness, de O'Leary y el CPOD, 4) se determinó la necesidad de atención odontológica, mediante registro de odontograma, y el estado de actividad y riego de enfermedad para determinar el tratamiento a realizar.

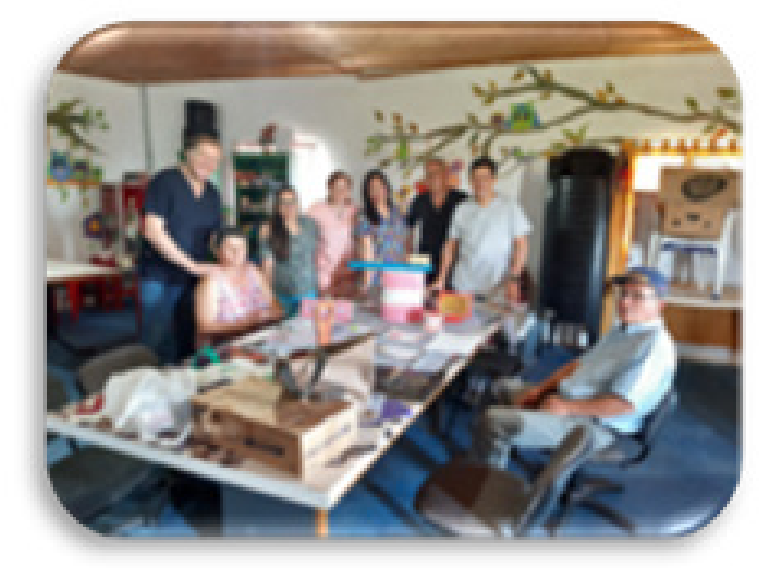

Figura 1. Equipo de extensionistas.
En el tercer módulo se registraron: 1) examen físico: peso talla, desarrollo de las características fenotípicas acorde al sexo y la edad, 2) control del biofilm dental, 3) inactivación de caries reduciendo los niveles de infección, 4) topicaciones con flúor para lograr el refuerzo del huésped, así como también control de nichos ecológicos por medio de aplicación de selladores de fosas y fisuras. Todas estas actividades se complementaron con la entrega de cartilla con información sobre dieta, salud general y bucal, con seguimiento a los 30 y 60 días.

\section{Resultados}

El grupo de extensión (Figura 1), trabajó con un total de 43 mujeres adolescentes, entre 13 y 22 años de edad, a las cuales se les brindó en un 100\% charlas educativas sobre cuidado en salud en general y bucal.

Se realizaron fichas médicas y odontológicas. En la ficha dental se dejó constancia del riesgo y actividad de caries, a través de la confección del odontograma.

En las diferentes visitas programadas, se realizó enseñanza de técnica de cepillado de Bass. En el $80 \%$ de las adolescentes, el Índice de biopelícula dental de O'Leary, arrojó un promedio de $20 \%$, compatible con salud.

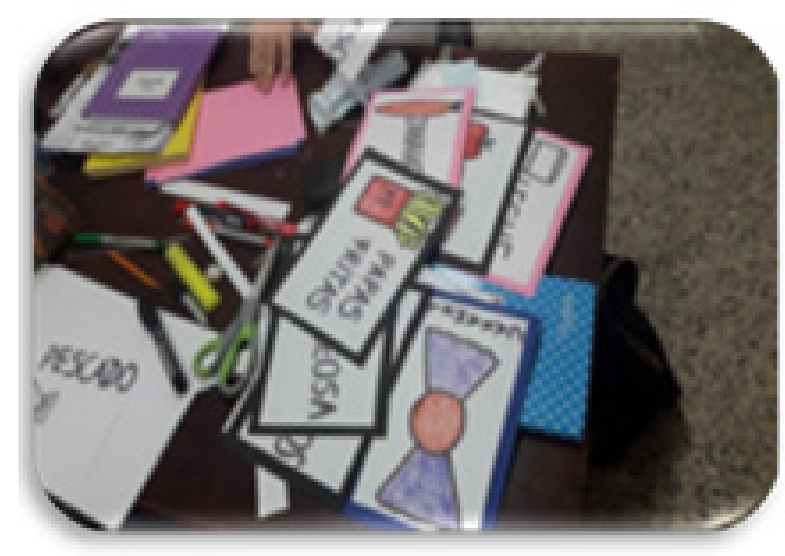

Figura 2. Cartillas informativas 
El índice de Loe y Silness que nos indica el estado de salud enfermedad de la encía, en los diferentes registros, obtuvo un valor de 1,5 indicando inflamación gingival leve.

También se logró disminuir los consumos frecuentes de azúcares, interviniendo con historias de dietas semanales, donde más del $60 \%$ logró modificar sus hábitos dietéticos (Figura 2).

\section{Conclusiones}

El trabajo interdisciplinario trajo una retroalimentación de conocimientos, en la que no solo se beneficia el destinatario, también los profesionales intervinientes, en este caso en mejorar la salud de los adolescentes.

Relacionar manifestaciones bucales y generales en salud, brinda herramientas para el autocuidado de mujeres adolescentes.

Teniendo presente que esta labor se realizó en comunidades con escasos recursos y pocas posibilidades de asistir en forma privada para su atención, buscamos dar las armas necesarias para lograr un cambio de conducta respectos a los hábitos de higiene y cuidado de la salud general y bucodental, y sobre todo que perdure en la conducta de las adolescentes.

\section{Referencias Bibliográficas}

1. Vaillant CM, Dandicourt TC, Mackensie SY. Prevención del embarazo en adolescentes. Rev Cubana Enfermer [Internet]. 2012 Jun [citado 02 oct 2014];28(2):125-35. Disponible en: http://scielo.sld.cu/scielo.php?script=sci_arttext\&pid=S0864192012000200008\&Ing=es

2. Rosabal García E, Romero Muñoz N, Gaquín Ramírez K, Hernández Mérida R. Conductas de riesgo en los adolescentes. Rev Cub Med Mil. 2015;44(2):218-229.

3. Subsecretaría de Prevención y Promoción de la Salud Centro Nacional de Programas Preventivos y Control de Enfermedades. Salud Bucal en la Adolescencia México, D.F. ISBN 978-607-460-325-5. $1^{\circ}$ edición, agosto de 2012. Disponible en: https://salud.edomex.gob.mx/isem/ documentos/temas_programas/sbucal/Manuales/MANUAL\%20SALUD\%20BUCAL\%20ADOLSESCENCIA.pdf 4. Font-Ribera L, García-Continente X, Davó-Blanes MC, Ariza C, Díez E, García- Calvente MM et al. El estudio de las desigualdades sociales en la salud infantil y adolescente en España. Gaceta Sanitaria. 2014;28(4):316-325. Disponible en: http://dx.doi.org/10.1016/j.gaceta.2013.12.009

5. Costa AC, Rodrigues FS, Heimer MV. La autopercepción de la estética dental y su impacto en la vida del adolescente. Adolesc Saude. 2017;14(4):157-166. Disponible en: http://www.adolescenciaesaude.com/detalhe_artigo. asp?id=696\&idioma $=$ Espanhol

6. Kelly Y, Zilanawala A, Sacker A, Hiatt R, Viner R. Early puberty in 11-year-old girls: Millennium Cohort Study findings. Arch Dis Child. 2017;102(3):232-237. DOI: http:// dx.doi.org/10.1136/archdischild-2016-310475

7. Cerón-Bastidas XA. Relación de calidad de vida y salud oral en la población adolescente Rev. CES Odont 2018;31(1):38-46. DOI: http://dx.doi.org/10.21615/cesodon.31.1.4

8. Crespo Cuenca L, Parra Enríquez S, Moreno Almaguer M, Matos Roche L, Aguilera Ochoa F. Intervención educativa sobre nivel de conocimientos en salud bucal en adolescentes de "Rafael Freyre". CCM. 2019;23(2):443-458. Disponible en: http://www.revcocmed.sld.cu/index.php/ cocmed/article/view/2667/1398 